\title{
A new fault diagnosis method of rotating machinery
}

\author{
Chih-Hao Chen ${ }^{\mathrm{a}, *}$, Rong-Juin Shyu ${ }^{\mathrm{a}}$ and Chih-Kao Ma ${ }^{\mathrm{b}}$ \\ ${ }^{a}$ Department of System Engineering and Naval Architecture, National Taiwan Ocean University, Keeling, Taiwan \\ ${ }^{\mathrm{b}}$ Graduate school of Gerontic Technology and Service Management, Nan Kai Institute of Technology, Tianjin, \\ Taiwan
}

Received 31 January 2007

Revised 2007

\begin{abstract}
This paper presents a new fault diagnosis procedure for rotating machinery using the wavelet packets-fractal technology and a radial basis function neural network. The faults of rotating machinery considered in this study include imbalance, misalignment, looseness and imbalance combined with misalignment conditions. When such faults occur, they usually induce non-stationary vibrations to the machine. After measuring the vibration signals, the wavelet packets transform is applied to these signals. The fractal dimension of each frequency bands is extracted and the box counting dimension is used to depict the failure characteristics of the vibration signals. The failure modes are then classified by a radial basis function neural network. An experimental study was performed to evaluate the proposed method and the results show that the method can effectively detect and recognize different kinds of faults of rotating machinery.
\end{abstract}

Keywords: Fault diagnosis, rotating machinery, wavelet packets, fractal, box counting dimension, radial basis function neural network

\section{Introduction}

Rotating machine faults detection and diagnosis are typically based on vibration measurements [1,2]. In general, the Fast Fourier Transform (FFT) is applied to obtain the power spectrum of the emitted vibration signals. The spectrum offers the frequency components of the time history signals. The spectral contents are analyzed to ascertain the operating conditions of the monitored machine. The spectrum of a vibration signal $x(t)$ can be obtained by the following equation:

$$
X(\omega)=\int_{-\infty}^{+\infty} x(t) * e^{i \omega t} d t
$$

From Eq. (1), every frequency component of the vibration signal will be displayed in its spectrum. Unfortunately, the rotating speed of the rotary machinery is hard to keep in a steady condition due to the factors such as loads, power supply, and faults. Then, the smearing effect will be generated a spectrum as shown in Fig. 1. In Fig. 1(b), the operating condition of a machine is worse. However, due to the amplitude and frequency modulations effect, the amplitude of the frequency component $79.1 \mathrm{~Hz}$ is smaller than that in Fig. 1(a). For this limitation of the FFT in analyzing non-stationary signals leads to the introduction of time-frequency or time-scale signal processing tools.

Recently, the wavelet packets transform has been widely applied to analyze non-stationary signals, and has proven effective in fault diagnosis of rotating machinery [3-7]. Wavelet packets transform is a time-frequency method,

\footnotetext{
* Corresponding author: Chih-Hao Chen, Department of System Engineering and Naval Architecture, National Taiwan Ocean University, 2 Pei-Ning Road, Keelung 20224, Taiwan. Tel.: +886 224622192 ext. 6033; Fax: +886 2246 24634; E-mail: d92510003@mail.ntou.edu.tw.
} 


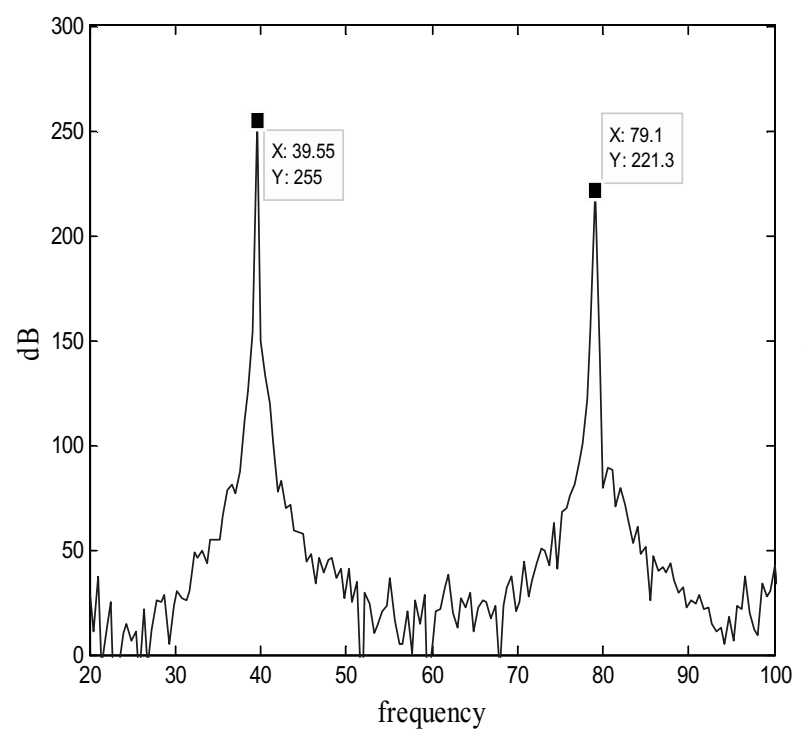

(a)

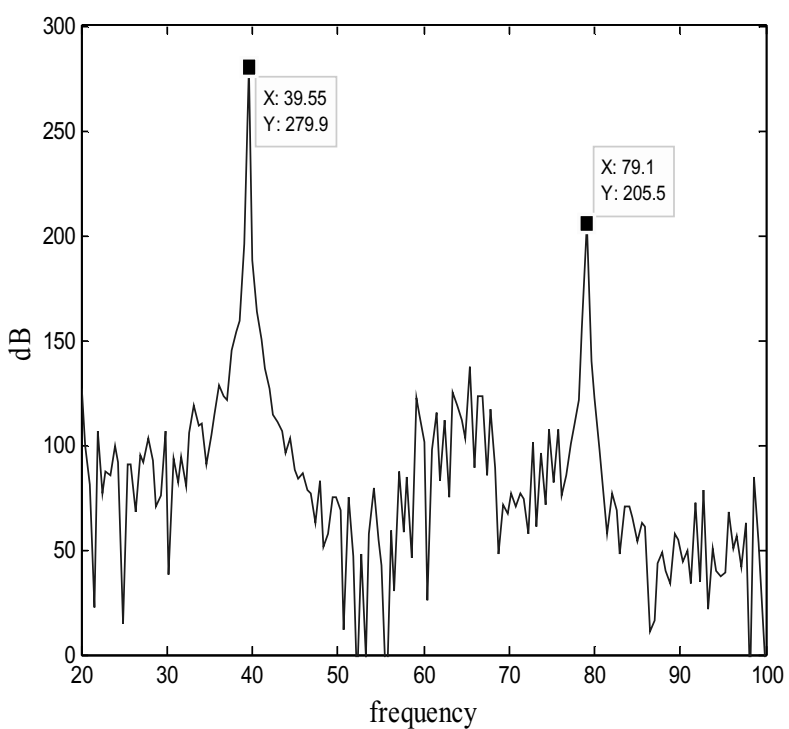

(b)

Fig. 1. The smearing effect in power spectrum.

which is a feature of multi-resolution analysis, and highlights localized signal characteristics in time and frequency domains simultaneously [8,9]. Thus, the signals can be presented from a global to specific time properties. Other key issues in fault diagnosis of rotating machinery include how to extract the valuable operating characteristics from the vibration signals and how to describe the non-stationary signals in a quantitative way that permits its evolution analysis.

Fractal refers to the fact that its geometric dimension is often a fraction rather than an integer [10]. It is able to describe some geometric objects that are not possible in traditional Euclidean geometry. Fractal was firstly introduced by Mandelbrot in his study of coastlines. Because of its universal existence [11,12], fractal analysis has been widely applied to many fields, including philosophy, economics, information, chemistry [13], medical science, and geography. Academics and experts use fractal theory to interpret varying and unpredictable physical phenomenon, and have achieved substantial results in image and signal processing [14]. But the application of fractal analysis to fault diagnosis in rotating machinery remains at its early stages.

Neural networks are widely used in the field of pattern recognition and classification because of their capacity to map non-linear functions [15]. Many types of networks have been successfully developed for rotating machinery fault classification. For example, Back Propagation (BP), Adaptive Resonance Theory (ART), Bidirectional Associative Memory (BAM), Brain State in a Box (BSB), Radial Basic Function (RBF) [16-18]. Among these, the BP network, a multi-layer feed forward network, is one of the best networks used in engineering applications. Many improved algorithms have been developed for BP networks to increase the speed of training and to avoid falling into local minimum during training. But their effectiveness in solving these problems is still not good enough. Furthermore, the determination of a suitable architecture for BP networks is difficult for inexperienced users, especially in terms of the number of neurons in the hidden layer $[19,20]$. The RBF network is a new and more effective training method, as it avoids complicated calculations and the time required for training is shorter than that of the BP network. Furthermore, it is capable of fast convergence and automatically determining the number of neurons in the hidden layer during training. Hence, in this study, we will use the RBF network to identify the faults of rotating machinery.

Fault diagnosis refers to the evaluation of the operating conditions of rotating machinery, based on measurements of dynamic signals, identification of the cause and position of the fault. In this paper, we investigated the following fault conditions, such as unbalance, misalignment, base looseness and unbalance combined with misalignment. After measuring the vibration signals, wavelet packets analysis is used to decompose vibration signals into a series 
of localized wavelet functions. Then, fractal theory is used to calculate the box-counting dimension of different frequency bands, and a structure fractal dimensions feature vector is established in a quantitative manner. Applying this vector as an input to a radial neural network, effective fault diagnosis model and fault model recognition can be established for rotating machinery. We used an experimental study to evaluate the performance of the proposed diagnosis method.

\section{Theory}

\subsection{Wavelet transform}

Wavelet transforms are particular linear combinations of wavelet functions called packets. They form an orthogonal bases that can retain many of the smoothness and time frequency localization properties of their corresponding wavelet. Moreover, the wavelet packet decomposition, which is an extension of the classical wavelet decomposition, offers a richer signal analysis and is a time-frequency function set $\left\{u_{n}(t)\right\}_{n \in Z}(Z=1,2,3, \ldots)$ which describes the information of function of $f(t)$ in various time windows and frequency bands as:

$$
s_{n}(t)=2^{-j} / 2 s_{n}\left(2^{j} t-k\right)
$$

The integer $j$ and $k$ are the index scale and the translation operations, respectively. The index $n=0,1, \ldots$ is called the modulation parameter. The first two wavelet packet functions are the scale and mother wavelet function, respectively:

$$
\begin{aligned}
& s_{0}(t)=\phi(t) \\
& s_{1}(t)=\psi(t)
\end{aligned}
$$

Wavelet packet functions for $n=1,2, \ldots$ are then defined by the following recursive relationships:

$$
\begin{aligned}
& h(t)=\frac{1}{2}\left\langle\phi\left[\frac{t}{2}\right], \phi[t-k]\right\rangle \quad t \in R, k \in Z \\
& g(t)=\frac{1}{2}\left\langle\psi\left[\frac{t}{2}\right], \psi[t-k]\right\rangle \\
& s_{2 n}(t)=\sqrt{2} \sum_{k \in Z} h(k) s_{n}(2 t-k) \\
& s_{2 n+1}(t)=\sqrt{2} \sum_{k \in Z} g(k) s_{n}(2 t-k)
\end{aligned}
$$

where $h(t)$ and $g(t)$ are the quadrature mirror filter bank associated with predefined scaling function and mother wavelet function. The wavelet packet coefficient of a function $f(t)$ can be computed via

$$
\text { coe } f=\int f(t) s_{n}(t) d t
$$

Because the wavelet packet basis is orthogonal, the coefficients of every frequency band represent the fractal dimension of signals in this frequency band. It is obvious that the features of the fault diagnosis of rotating machinery can be obtained by using these coefficients. But not all frequency bands are available. We should choose the most important features that can reflect the fault conditions of rotating machinery. The wavelet packet decomposition tree is produced as shown in Fig. 2.

\subsection{Fractal theory}

Fractal dimension is an important parameter to describe fractal characteristics in quantitatively and can be used to describe the complexity of signals. In general, different signals contain different fractal dimensions. Thus, fractal dimensions can effectively extract the characteristics of signals. Fractal dimensions have been developed into more than a dozen different dimensions, including Hausdorff, box counting, capacity, correlation, and Lyapunov dimensions. The calculation of the box counting dimension is relatively simple. Therefore, after using the wavelet 


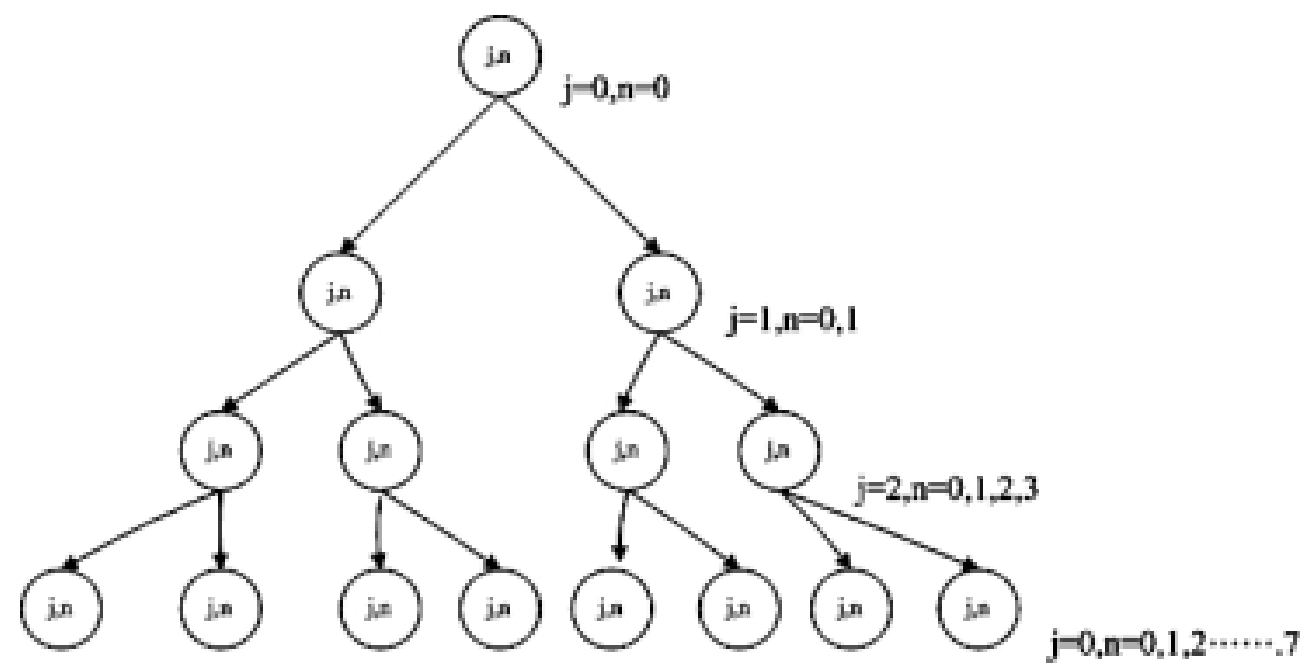

Fig. 2. Wavelet packets decomposition tree.

packets decomposition, this study will use it to analyze the fractal phenomenon of vibration signals in different frequency bands.

The algorithm of the box counting dimensions assumes a time serial signal $s(j) \subset S$, where $S$ is a closed set $R^{n}$ in $n$ dimension Euclidean space. Divide $R^{n}$ into a very fine grid and if $N_{r}$ is the number of grids in a discrete space set of size $r$, then the box counting dimension is defined as:

$$
d_{B}=\lim \left(-\frac{\log \left(N_{r}\right)}{\log (r)}\right)
$$

Since the highest recognition rate of time series is $s(j)$, the sampling interval of $r$ is unable to approach zero. In the actual calculation it will generally be an approximate algorithm and gradually enlarge grid $r$ to be grid $k_{r}$, where $k=1,2, \ldots$. Then $N_{r}$ can be calculated by using the following formulas:

$$
P\left(k_{r}\right)=\sum_{j=1}^{N_{0} / K}\left|\max \left\{\begin{array}{l}
s_{k(j-1)+1}, s_{k(j-1)+2}, \ldots \\
\ldots, s_{k(j-1)+k+1}
\end{array}\right\}-\min \left\{\begin{array}{l}
s_{k(j-1)+1}, s_{k(j-1)+2}, \ldots \\
\ldots, s_{k(j-1)+k+1}
\end{array}\right\}\right|
$$

where $\begin{aligned} j & =1,2, \ldots, \frac{N_{0}}{K} \\ k & =1,2, \ldots, K, K<N_{0}\end{aligned}$

$$
N_{r}=P\left(k_{r}\right) /\left(k_{r}\right)+1 \quad N_{r}>1
$$

The grid number $N_{r}$ and grid width $k_{r}$ can produce a double logarithm curve $\left(\log \left(N_{r}\right)-\log \left(k_{r}\right)\right)$. Since the fractal phenomenon is not always present. We can use the $\left(\log \left(N_{r}\right)-\log \left(k_{r}\right)\right)$ curve to check the existence of a scale-invariant region.

We used the least square method to assure the linear regression is a straight line. The box counting dimension is the slope of the straight line. The fractal size of a curve is determined by the range occupied by the curve in the space. We used a straight line $f(x)=2 x+3$ as a test example. The size of the fractal dimension of this line is equal to topology dimension 1.0. The estimated result from the box counting dimension logarithm is 1.0418, as shown in Fig. 3. The results show that the algorithm of the box counting dimension can accurately predict the fractal dimension of this curve.

\subsection{Radial basis function neural network}

The RBF Neural Network is a feedforward network. Its architecture consists of three layers, i.e., the input layer with $\mathrm{R}^{*} 1$ input neurons, a hidden radial basis layer of $\mathrm{S}^{1} * \mathrm{R}$ neurons, and an output linear layer of $S^{2} * 1$ neurons, 


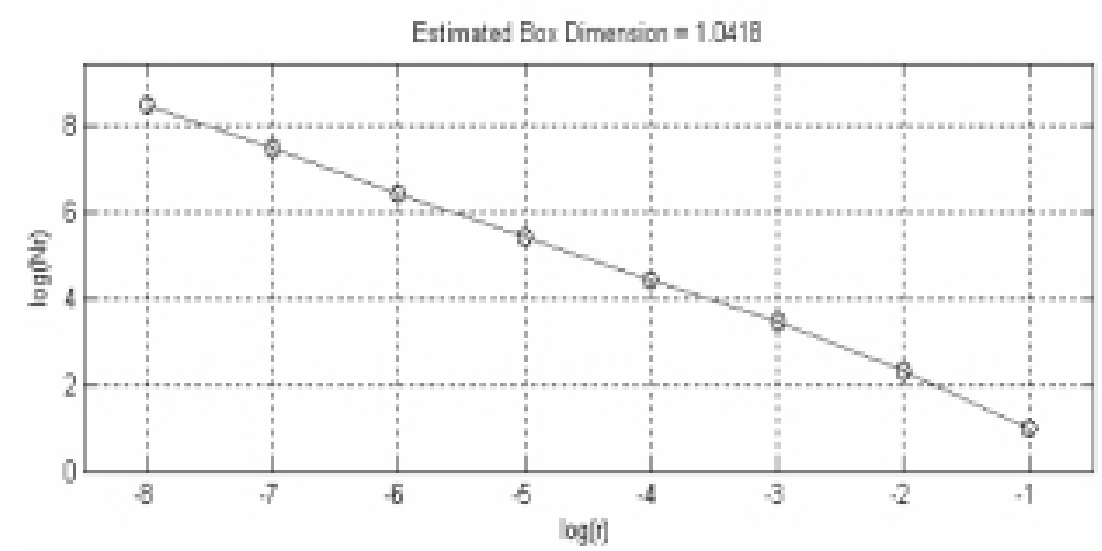

Fig. 3. Straight line box counting dimension estimation result.
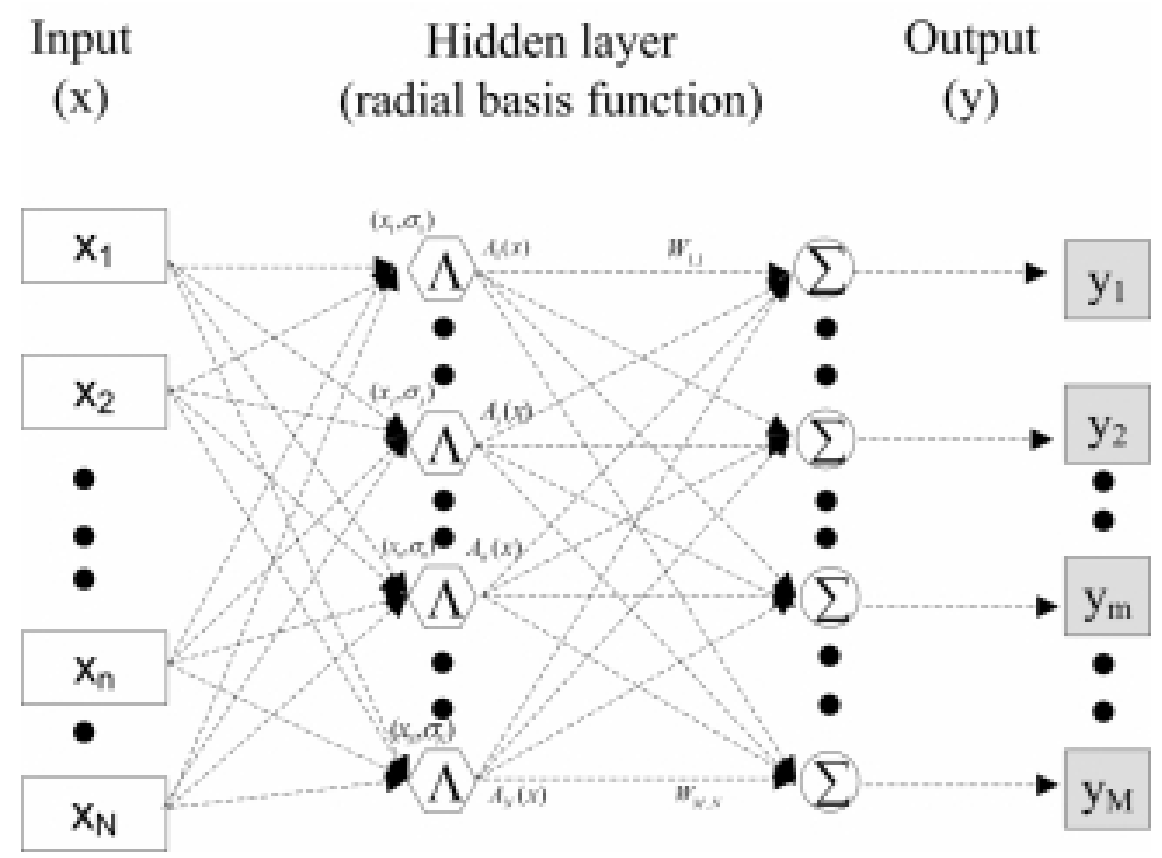

Fig. 4. Structure of RBF neural network.

as shown in Fig. 4. In the RBF neural network, the transfer function of the hidden layer is a Gaussian function as follows:

$$
A_{j}=\exp \left(\frac{-\left|x-x_{j}\right|^{2}}{2 \sigma_{j}^{2}}\right), \quad j=1,2, \ldots, N
$$

where $A_{j}$ is the output of the $\mathrm{j}^{\text {th }}$ neuron in the hidden layer, $x$ is the input mode, $x_{j}$ is the center of the $\mathrm{j}^{\text {th }}$ neuron Gaussian function, $\sigma_{j}^{2}$ is the unitary parameter, and $j$ is the number of hidden nodes. The output of the RBF neuron network linear layer is given by:

$$
y_{k}=W_{k, j}^{T} A_{k}, \quad k=1,2, \ldots, M
$$




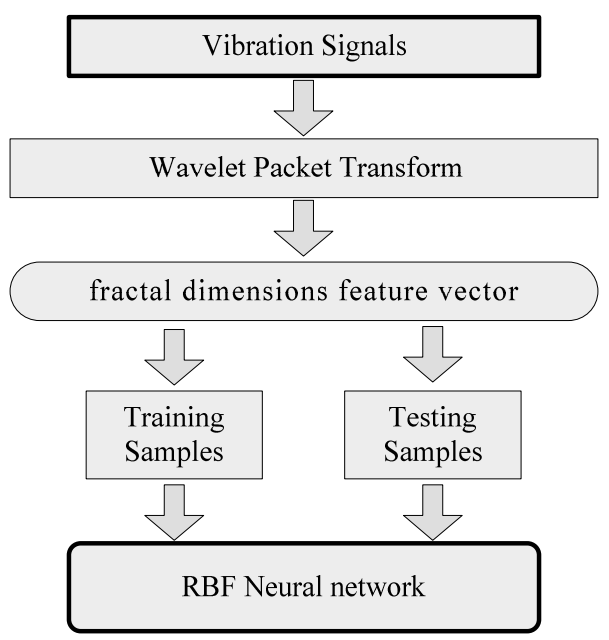

Fig. 5. The fault diagnosis procedure of rotating machinery.

where $y_{k}$ is output of the $k^{t h}$ node, $W_{k, j}$ is weight vector for node $k$ and $A_{k}$ is output vector from the $k^{t h}$ hidden layer. Each neuron of the RBF neural network output is a value that depends on its weight from the center of the neural network.

In this study, the RBF neural network is used for the fault recognition architecture. The fault characteristics and fault cause are the nodes of input layer and output layer, respectively. Therefore, for the network learning and training, the input vector is the response of the fault phenomena and the output vectors are the corresponding fault causes. Based on the learning and training of such samples, the network structure is adjusted to be the recognition tool in the fault diagnosis of rotating machinery.

This paper presents a new fault diagnosis approach using wavelet fractal techniques and neural networks. The procedure of this approach is illustrated in Fig. 5. After measuring the vibration signals, the first step is to conduct the wavelet packets decomposition and categorize the results according to each section of a perpendicular frequency band. The next step is to calculate the box counting dimension of each frequency bands and use the results to generate a feature vector set, which provides the input for the RBF neural network. Using this learning and training of network, the fault causes can then be identified.

\section{Experimental investigation and discussion}

\subsection{Experimental set-up}

The experimental set-up is shown in Fig. 6. In this study, we considered 12 faults condition of unbalance, misalignment and base looseness as shown in Table 1.

We used an accelerometer (Bruel \& Kjaer 4384) with an charge amplifier (Bruel \& Kjaer 2635) to measure the vibration signals of the rotor, as shown in Fig. 7. The signals were recorded on magnetic tapes (SONY Pca 208A), digitalized through an acquisition unit (National Instrument Daq-1200), and then analyzed using the algorithm developed by this study. The maximum analysis frequency was set at $1000 \mathrm{~Hz}$, and the sampling frequency was $2560 \mathrm{~Hz}$ in all the investigated cases. The rotation speed of the motor was set at $2400 \mathrm{rpm}\left(f_{r}=40 \mathrm{~Hz}\right)$. In these experiments were collected 35 groups of vibration signals by fault condition. From these groups of signals 20 groups were used as training samples to train the RBF neural network. The rest 15 groups were used as samples to test the recognition ability of the neural network. 
Table 1

Faults condition hypothesis steps

\begin{tabular}{lll}
\hline Fault condition & Quantitative description & Qualitative description \\
\hline Condition A & Use a rotor balancer (GT 5052A, G-TECH) to balance the rotor. & Normal condition \\
Condition B_1 & Put a grams screw on the adjustable disc & Unbalance of the initial stage \\
Condition B_2 & Put a grams screw on the adjustable disc & Unbalance of the bad stage \\
Condition B_3 & Put a grams screw on the adjustable disc & Unbalance of the worse stage \\
Condition C_1 & Insert one sheet of copy paper into the adjustable disc. & Misalignment of the initial stage \\
Condition C_2 & Insert two sheet of copy paper into the adjustable disc. & Misalignment of the bad stage \\
Condition C_3 & Insert three sheet of copy paper into the adjustable disc. & Misalignment of the worse stage \\
Condition D_1 & Loose one of the four screws of bearing base to 360 degrees. (one circle) & The base looseness of the initial stage \\
Condition D_2 & Loose one of the four screws of bearing base to 720 degrees. (two circles) & The base looseness of the bad stage \\
Condition D_3 & Loose one of the four screws of bearing base to 1080 degrees. (three circles) & The base looseness of the worse stage \\
Condition E_1 & & Combination of the condition B_1 and C_1 \\
Condition E_2 & & Combination of the condition B_2 and C_2 \\
\hline
\end{tabular}

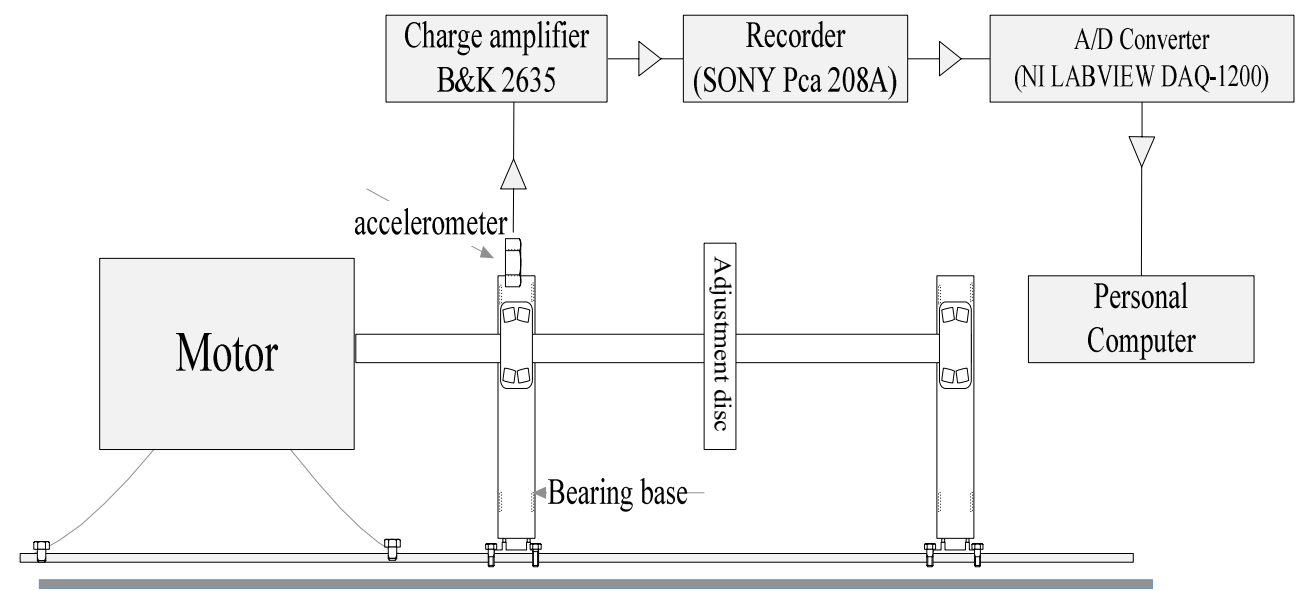

Fig. 6. Experimental set-up.

\subsection{Power spectrum}

Most of the frequency domain signal-processing methods were based on the assumption that signals are stationary. However, in this study the smearing effect was found in the power spectrum of the measured signals. For example, Fig. 8(a) shows the result of the fault condition C_1, where the amplitude of frequency $2 f_{r}$ is $129.8 \mathrm{~dB}$. As the condition changes to $\mathrm{C} 2$, the amplitude of $2 f_{r}$ increases to $182.2 \mathrm{~dB}$, as shown in Fig. 8(b). When the fault condition changes to $\mathrm{C} 3$, the amplitude decreases to $98.72 \mathrm{~dB}$, due to the smearing effect, as shown in Fig. 8(c). That means, for non-stationary signals, the effect of smearing spreads the energy of the signal over a frequency band. Therefore, in this study, we cannot use the amplitude of a frequency component in the power spectrum to diagnose the faults of rotating machinery.

\subsection{Wavelet packets-fractal}

After extracting the vibration signals of different fault conditions, the raw signals were examined to see if they contained fractal phenomenon. Following a box counting dimension calculation of the raw signals, fractal logarithm curves were obtained, as shown in Fig. 9. The figure reveals that each curve has a major linear (some graphics shown two linear segment) segment at the scale-invariant region. This phenomenon confirms the measured vibration signals are suitable for the application of fractal analysis. A comparison of the measured box counting dimensions of different fault conditions, was indicated in Fig. 10. In the figure, it is observed that:

1. Normal condition: dimension values range from 1.1 to 1.23. 

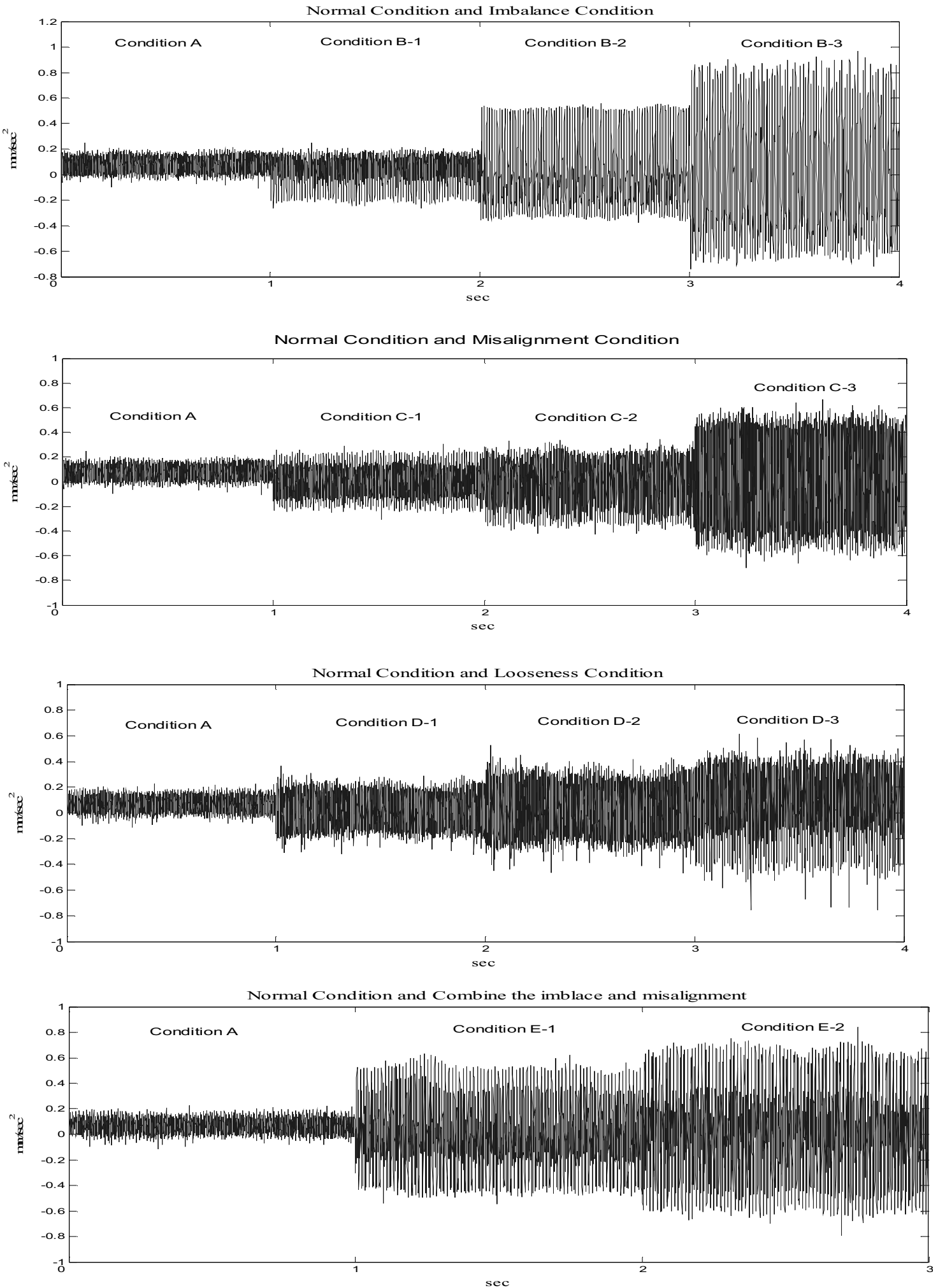

Fig. 7. Vibration signals in time domain. 


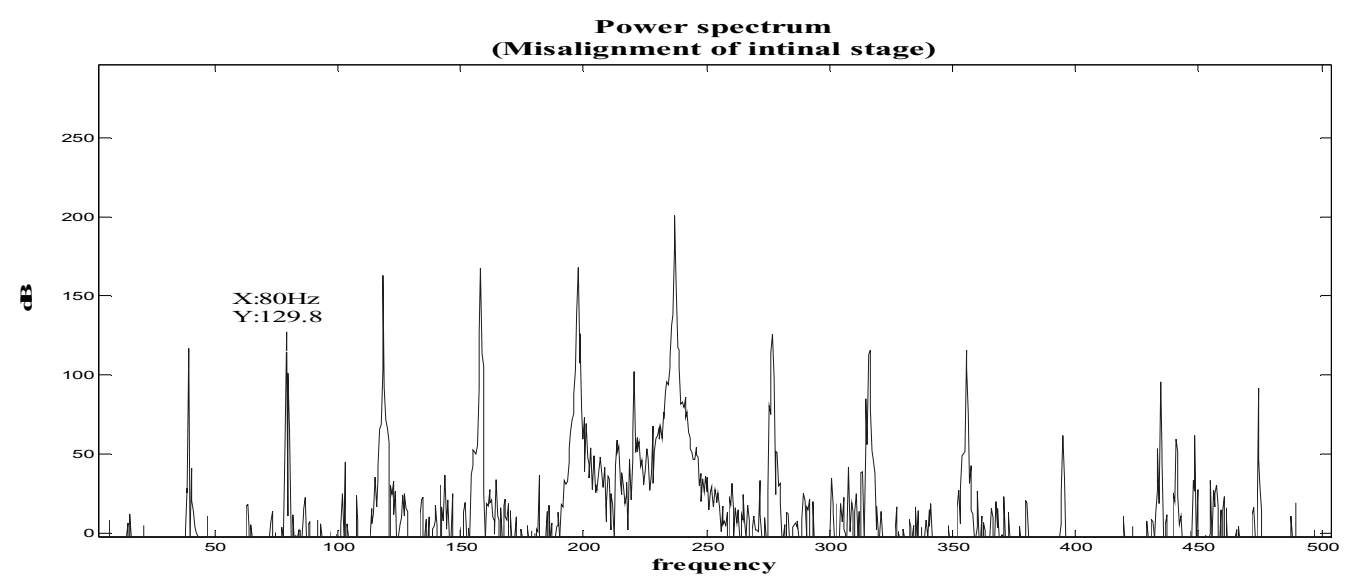

(a)

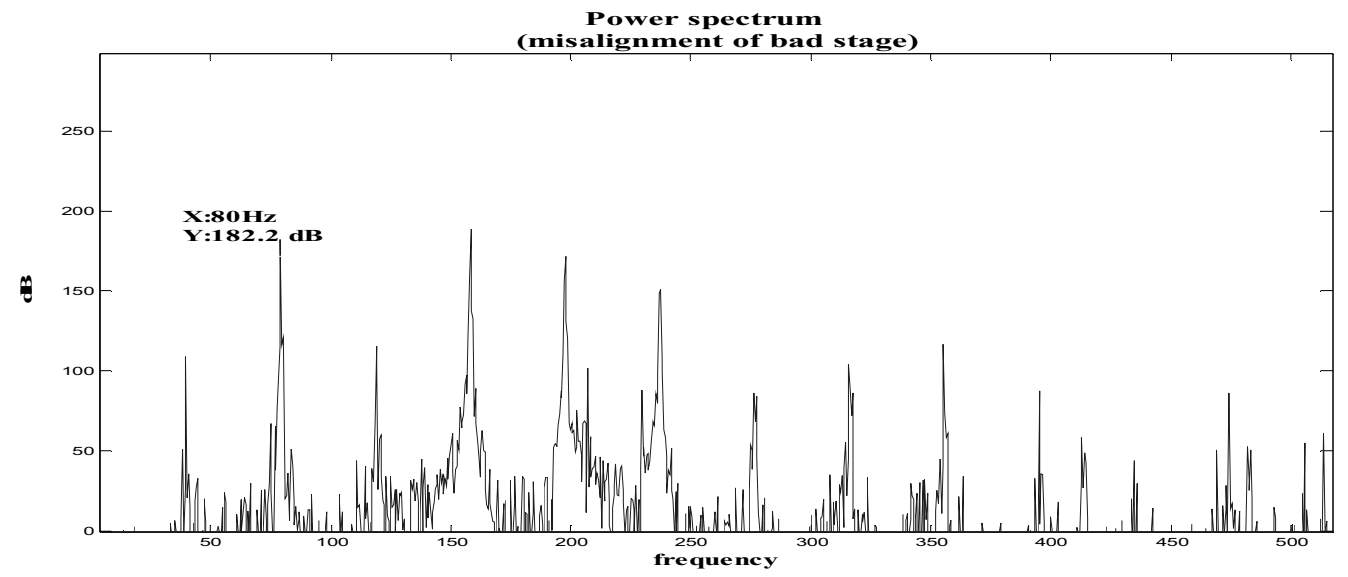

(b)

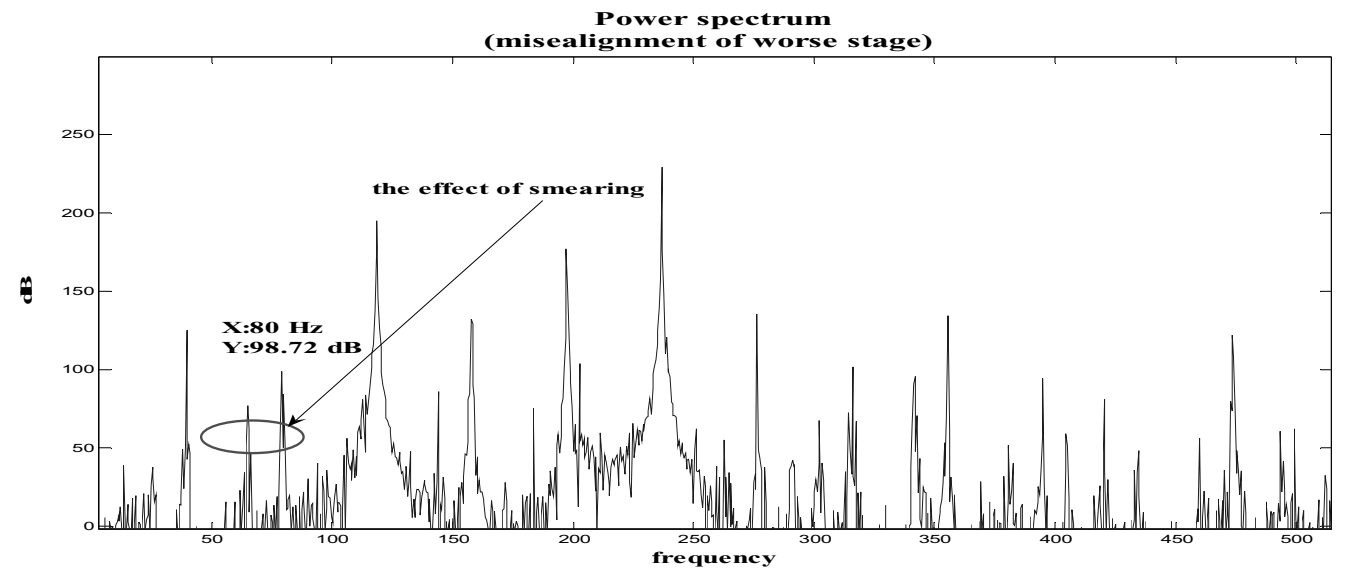

(c)

Fig. 8. The smearing effect in the power spectrum of fault condition C_3. 

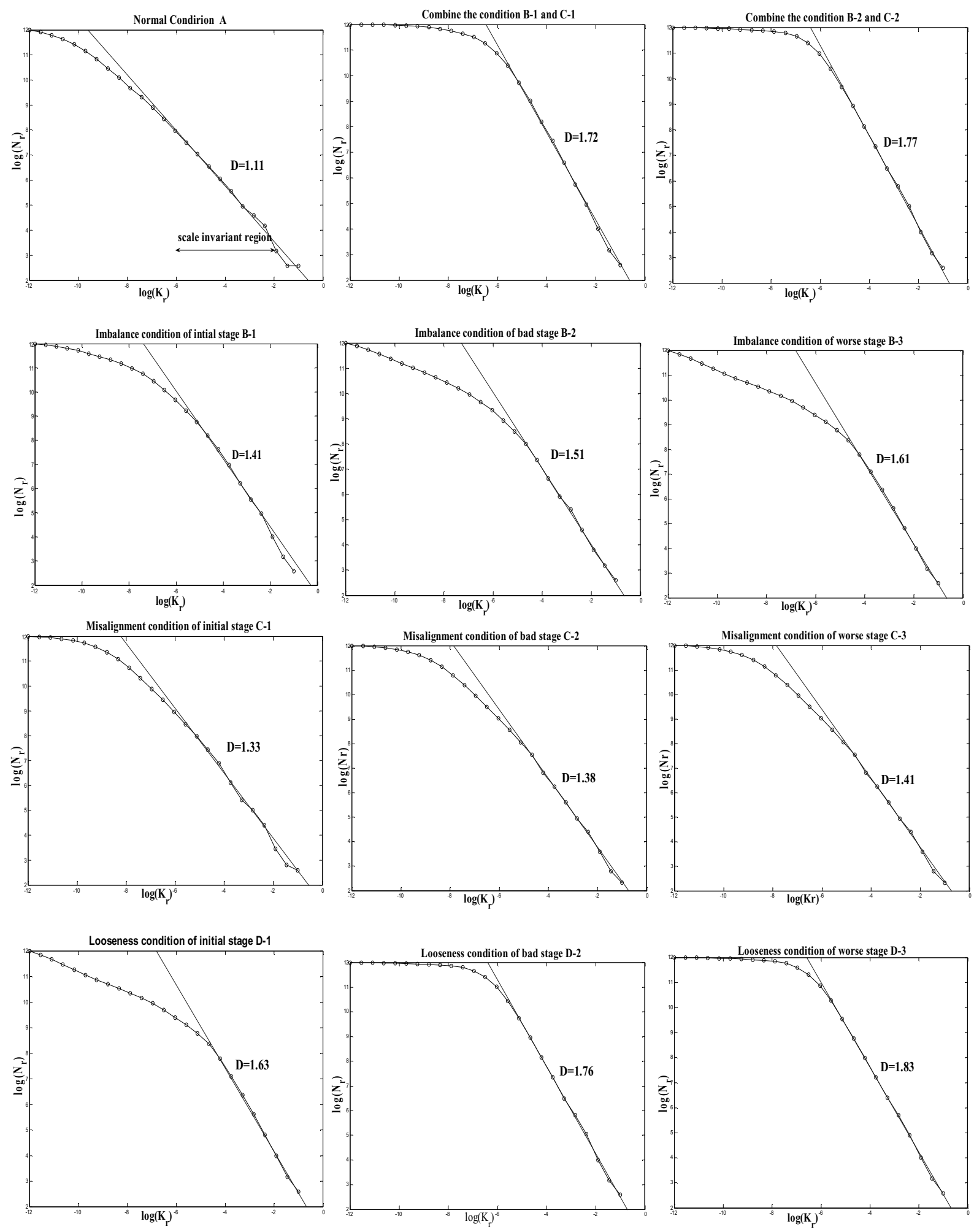

Fig. 9. The fractal logarithm curves of different fault conditions 


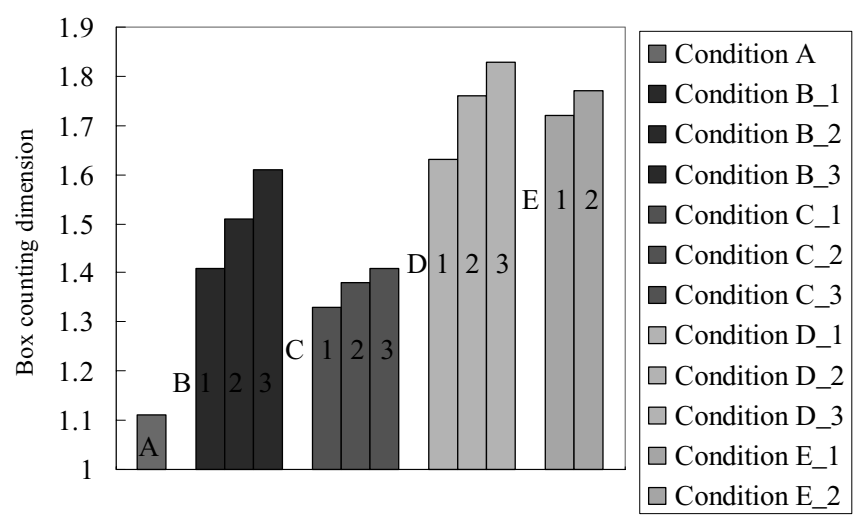

Fig. 10. Box counting dimension estimation results.

2. Unbalance fault (condition B_1, B 2 2, and B 3 3): dimension values range from 1.42 to 1.63 .

3. Misalignment fault (condition $C_{-} \_1, C_{2} 2$, and $C \_3$ ): dimension values range from 1.34 to 1.42 .

4. Base looseness fault (condition D_1, D 2 2, and D 3): dimension values range from 1.65 to 1.83 .

5. Combination of the condition $\mathrm{B}$ and $\mathrm{C}$ (condition $\mathrm{E} 1$ and $\mathrm{E} 2$ ): dimension values range from 1.71 to 1.76.

The results indicate that the box counting dimension values vary with fault conditions. The dimension value of the normal condition is the smallest, while fault condition D 3 is the largest. Also, the dimension value of the combined fault conditions is larger than that of each separate fault condition.

To extract the characteristics of each fault condition, wavelet packets transformation was used to divide the measured vibration signals into five levels using Coiflet 3-order wavelet function. Each signal is divided into 32 frequency bands, and each band width is $31.25 \mathrm{~Hz}$. This band width can avoid the rotating frequency $f_{r}$ and its harmonic frequencies existing simultaneously in the same frequency band. The results of extracting the box counting dimensions of the signals in each frequency band show the changes of dimension values of different fault conditions. The obvious dimension changes in specific frequency bands of different fault conditions are summarized in Fig. 11. The dimension change of fault condition B_1, B 2 and B 3 occurred in the second and fourth frequency bands, i.e., $f_{r}$ and $2 f_{r}$. Fault condition $\mathrm{C}_{-} 1, \mathrm{C}_{2} 2$, and $\mathrm{C}_{-} 3$ had most prominent dimension changes in the third and fifth frequency bands. A dimensional characteristics of fault condition D 1, D 2, and D 3 were found in the third, fourth and seventh frequency bands and fault condition E 1 and E 2 in the second, fourth, sixth and eleventh frequency bands. These results are consistent with the investigation described in prior study [21]. Hence, in this study, we only calculated the box counting dimensions of the nine frequency bands i.e., $(0,1,2,3,4,7,8,10$ and 11) and used the results as a set of characteristic vectors. The vectors are used as the input of RBF neural network for learning and recognition.

\subsection{RBF neural network learning and testing}

The box counting dimension characteristic vector was normalized to be within 0 and 1 as the input neurons of the RBF neural network. Each input vector has ten elements, ten nodes in hidden layer, twelve nodes in output layer. The training precision was set to $10^{-6}$. Table 1 shows the training and learning results of the RBF neural network. From Table 1, we can conclude that the characteristics of all fault conditions have been successfully extracted from the measured vibration signals by using wavelet packet-fractal technology. Also, the training results of the RBF neural network for all fault condition are accurate.

Finally, the test samples were put into the trained fault recognition system to identify the type of fault. The results are shown in Table 2. The accuracy of all recognitions is over $73 \%$. To increase the accuracy of recognitions, one can use more effective samples to train the system. 

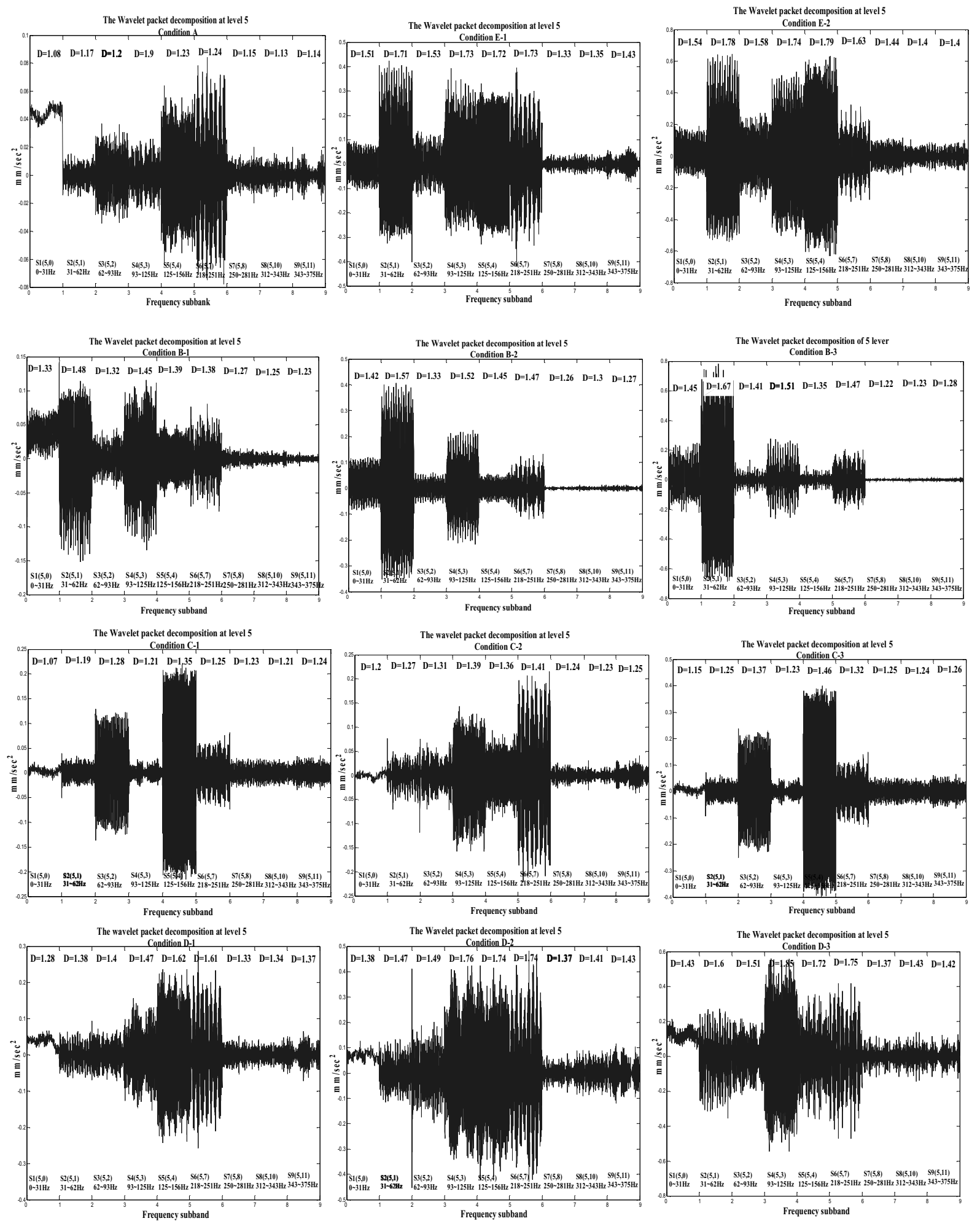

Fig. 11. The wavelet packet decomposition results 
Table 2

Results of RBF-NN training and learning

\begin{tabular}{|c|c|c|c|c|c|c|c|c|c|c|c|c|}
\hline \multirow{2}{*}{$\begin{array}{l}\text { Training } \\
\text { sample }\end{array}$} & \multicolumn{10}{|c|}{$\begin{array}{c}\text { RBF-NN input layer } \\
\text { Box counting dimension characteristic vector (normalized) }\end{array}$} & \multirow{2}{*}{$\begin{array}{l}\text { Fault } \\
\text { types }\end{array}$} & \multirow{2}{*}{$\begin{array}{c}\text { Training } \\
\text { result }\end{array}$} \\
\hline & $\begin{array}{c}\text { Original } \\
\text { signal }\end{array}$ & $\mathrm{S} 1(5,0)$ & $\mathrm{S} 2(5,1)$ & $\mathrm{S} 3(5,2)$ & $\mathrm{S} 4(5,3)$ & $\mathrm{S} 5(5,4)$ & S6 $(5,7)$ & $\mathrm{S} 7(5,8)$ & $\mathrm{S} 8(5,10)$ & S9(5,11) & & \\
\hline 1 & 0.25 & 0.1 & 0.55 & 0.7 & 0.65 & 0.85 & 0.9 & 0.45 & 0.35 & 0.4 & $\mathrm{~A}$ & $\mathrm{~A}$ \\
\hline 2 & 0.68 & 0.42 & 0.9 & 0.39 & 0.8 & 0.61 & 0.58 & 0.228 & 0.16 & 0.1 & B_1 & B_1 \\
\hline 3 & 0.75 & 0.51 & 0.9 & 0.28 & 0.77 & 0.59 & 0.64 & 0.1 & 0.2 & 0.13 & B_2 & B_2 \\
\hline 4 & 0.79 & 0.51 & 0.9 & 0.44 & 0.62 & 0.33 & 0.54 & 0.1 & 0.12 & 0.2 & B_3 & B_3 \\
\hline 5 & 0.9 & 0.1 & 0.47 & 0.75 & 0.53 & 0.96 & 0.65 & 0.59 & 0.53 & 0.62 & C_1 & C_1 \\
\hline 6 & 0.86 & 0.1 & 0.4 & 0.56 & 0.9 & 0.77 & 0.98 & 0.27 & 0.23 & 0.31 & C__2 & C__2 \\
\hline 7 & 0.77 & 0.1 & 0.36 & 0.67 & 0.31 & 0.9 & 0.54 & 0.36 & 0.33 & 0.38 & C_3 3 & C_3 \\
\hline 8 & 0.9 & 0.1 & 0.33 & 0.37 & 0.53 & 0.88 & 0.85 & 0.21 & 0.24 & 0.3 & D_1 & D_1 \\
\hline 9 & 0.9 & 0.1 & 0.29 & 0.33 & 0.9 & 0.86 & 0.86 & 0 & 0.16 & 0.21 & D_2 & D_2 \\
\hline 10 & 0.9 & 0.12 & 0.45 & 0.28 & 0.94 & 0.69 & 0.74 & 0 & 0.12 & 0.1 & D_3 & D_3 \\
\hline 11 & 0.88 & 0.46 & 0.86 & 0.5 & 0.9 & 0.88 & 0.9 & 0.1 & 0.14 & 0.3 & E_1 & E_1 \\
\hline 12 & 0.86 & 0.39 & 0.88 & 0.47 & 0.8 & 0.9 & 0.57 & 0.18 & 0.1 & 0.1 & E_2 & E_2 \\
\hline
\end{tabular}

Table 3

Accuracy of fault recognitions

\begin{tabular}{cccc}
\hline $\begin{array}{c}\text { Test } \\
\text { sample }\end{array}$ & $\begin{array}{c}\text { Number of } \\
\text { inputs }\end{array}$ & $\begin{array}{c}\text { Number of correct } \\
\text { recognitions }\end{array}$ & $\begin{array}{c}\text { Accuracy of fault recognitions } \\
\text { (number of correct recognitions } \text { /number of inputs) }\end{array}$ \\
\hline A & 15 & 13 & $86.7 \%$ \\
B_1 & 15 & 12 & $80 \%$ \\
B_2 & 15 & 15 & $100 \%$ \\
B_3 & 15 & 13 & $86.7 \%$ \\
C_1 & 15 & 15 & $100 \%$ \\
C_2 & 15 & 12 & $80 \%$ \\
C_3 & 15 & 11 & $73 \%$ \\
D_1 & 15 & 13 & $86.7 \%$ \\
D_2 & 15 & 15 & $100 \%$ \\
D_3 & 15 & 15 & $100 \%$ \\
E_1 & 15 & 11 & $73 \%$ \\
E_2 & 15 & 13 & $86.7 \%$ \\
\hline
\end{tabular}

\section{Conclusion}

This paper proposes a new fault diagnosis method of rotating machinery which integrates wavelet packets fractal techniques and RBF neural networks. The wavelet packets decomposition can accurately decompose vibration signals into various perpendicular frequency bands without losing the characteristics of the measured vibration signals. Also, fractal theory is used to extract the fault characteristics of various frequency bands and construct box counting dimension vectors to represent the fault conditions. Finally, a RBF neural network is used to perform the learning and recognitions of fault condition.

The results of the experimental investigation demonstrate the effectiveness of present diagnosis procedure for extracting the characteristics and recognizing the types the fault conditions of rotating machinery. Future works of this study will include the application of real rotating machines and the effectiveness of diagnosis for other types of fault conditions.

\section{Nomenclature}

$$
\begin{array}{ll}
\mathrm{x}(\mathrm{t}) & \text { vibration signal } \\
n=0,1, \ldots & \text { modulation parameter }
\end{array}
$$




$\begin{array}{ll}h(t), g(t) & \text { quadrature mirror filter bank } \\ \phi(t) & \text { scaling function } \\ \psi(t) & \text { mother wavelet function } \\ N_{r} & \text { number of grids } \\ r & \text { grid of size } \\ d_{B} & \text { box counting dimension } \\ s(j) & \text { time series } \\ A_{j} & \text { Gaussian function } \\ \sigma_{j}^{2} & \text { unitary parameter } \\ W_{k, j} & \text { weight vector }\end{array}$

\section{References}

[1] R.H. Lyon, Machinery Noise and Diagnostics, Massachusetts Institute of Technology, Butterworth Publishers, USA, 1987.

[2] N. Tandon, and A. Choudhury, A Review of Vibration and Acoustic Measurement Methods for The Detection of Defects in Rolling Element Bearings, Tribology International 32(8) (1999), 469-480.

[3] D. Boulahbal, M.F. Golnaraghi, F. Ismail, Amplitude and Phase Wavelet Maps for the Detection of Cracks in Geared Systems, International Journal of Mechanical Systems and Signal Processing 13(3) (1999), 423-436.

[4] Z. Geng and L. Qu, Vibrational Diagnosis of Machine Parts Using the Wavelet Packet Technique, British Journal of Non-Destructive Testing 36(1) (1994), 11-15.

[5] S. Lu, W. Chen and M. Li, Fault Pattern Recognition of Rolling Bearing Based on Wavelet Packet and Support Vector Machine, The Sixth World Congress on Intelligent Control and Automation 2 (2006), 5516-5520.

[6] B. Liu, Selection of Wavelet Packet Basis for Rotating Machinery Fault Diagnosis, Journal of Sound and Vibration 284 (2005), $567-582$.

[7] H. Zhang, S.J. Wang and Q.S. Zhang, The Research on Rolling Element Bearing Fault Diagnosis Based on Wavelet Packets Transform, Industrial Electronics Society, The 29th Annual Conference of the IEEE 2 (2003), 1745-1749.

[8] S. Mallat, A Wavelet Tour of Signal Processing, Academic Press, San Diego, CA, USA, 1998.

[9] W.J. Wang, Wavelets for Detecting Mechanical Faults with High Sensitivity, Mechanical Systems and Signal Processing 15(4) (2001), 685-696.

[10] J.L. McCauley, Chaos, dynamics, and fractals: An Algorithmic Approach to Deterministic Chaos, Cambridge Nonlinear Science Series 2, USA, 1993.

[11] B.B. Mandelbrot, Fractals: from, chance and dimension, Freeman, San Francisco, CA, New York, 1977.

[12] B.B. Mandelbrot, The Fractal Geometry of Nature, Freeman, San Francisco, CA, New York, 1983.

[13] C. Huang and H.B. Shi, Study on Chemical Process Faults Diagnosis Based on Fractal Geometry, Proceedings of the 5th World Congress on Intelligent Control and Automation, Hangzhou, China, 2, 2004, 1658-1662.

[14] D.C. Popescu, A. Dimca and H. Yan, A Nonlinear Model for Fractal Image Coding, IEEE Transactions on Image Processing 6(3) (1997), $373-382$.

[15] A.M. Arbib, The Handbook of Brain Theory and Neural Networks, Second Edition, Massachusetts MIT Press, 2002.

[16] M. Catelani and A. Fort, Fault diagnosis of electronic analog circuits using a radial basis function network classifier, Measurement 28 (2000), 147-158.

[17] S. Chen, C.F.N. Cowan and P.M. Grant, Orthogonal least squares learning algorithm for radial basis function networks, IEEE Trans on Bio Eng 2 (1991), 302-309.

[18] W. Lai, P.W. Tse, G. Zhang and T. Shi, Classification of gear faults using cumulants and the radial basis function network, Mechanical Systems and Signal Processing 18(2) (2004), 381-389.

[19] C. Fan, Z. Jin, J. Zhang and W. Tian, Application of Multisensor Data Fusion Based on RBF Neural Networks for Fault Diagnosis of SAMs, Seventh International Conference on Control, Automation, Robotics and Vision 3 (2002), 1557-1562.

[20] S.S. Haykin, Neural Networks: A Comprehensive Foundation, 2nd ed., Prentice Hall, Upper Saddle River, NJ, USA, 1999.

[21] G. Betta, C. Liguori, A. Paolillo and A. Pietrosanto, A DSP-Based FFT-Anlyzer for the fault diagnosis of rotating machine based on vibration analysis, IEEE Transactions on Instrumentation and Measurement 51(6) 1316-1322. 

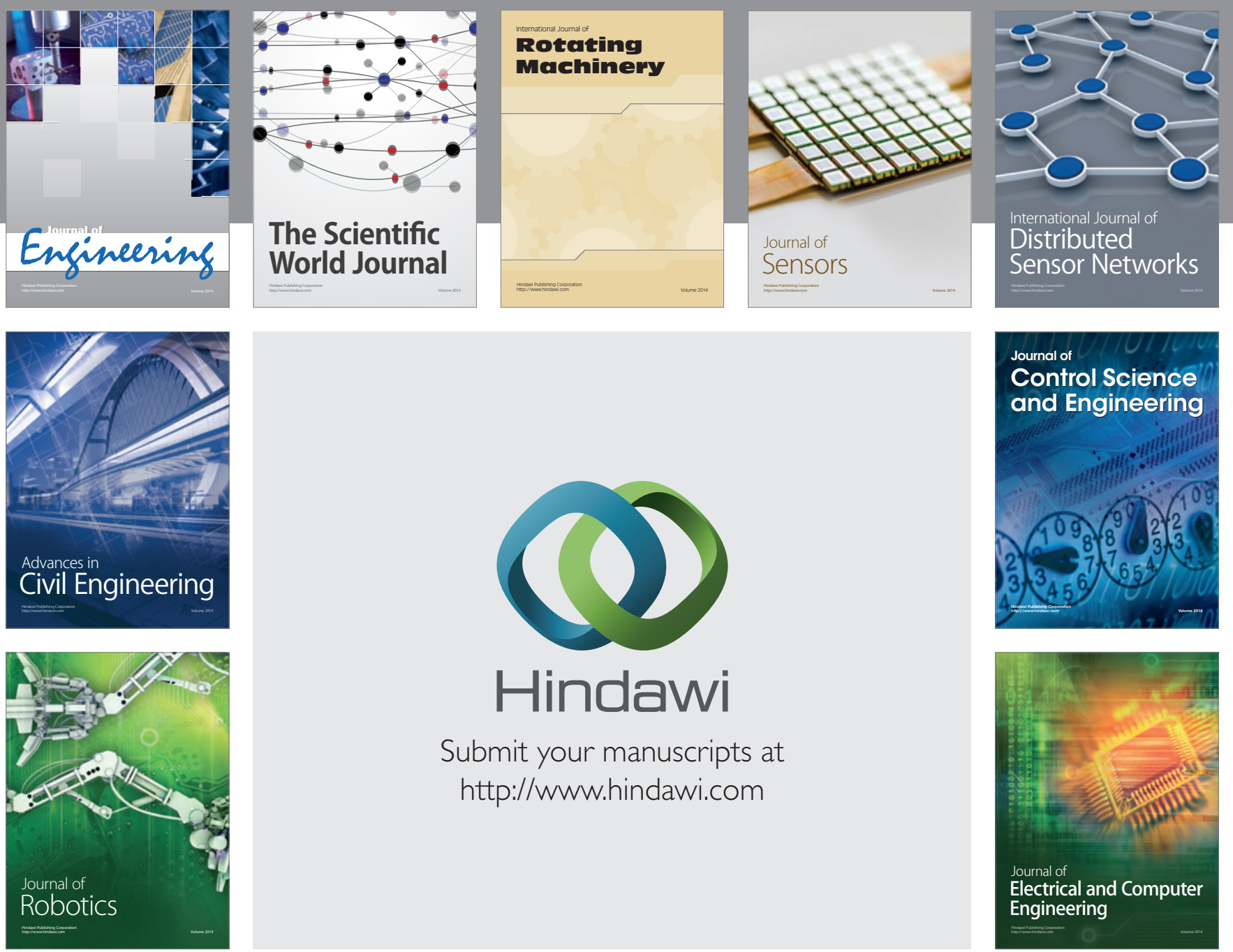

Submit your manuscripts at

http://www.hindawi.com
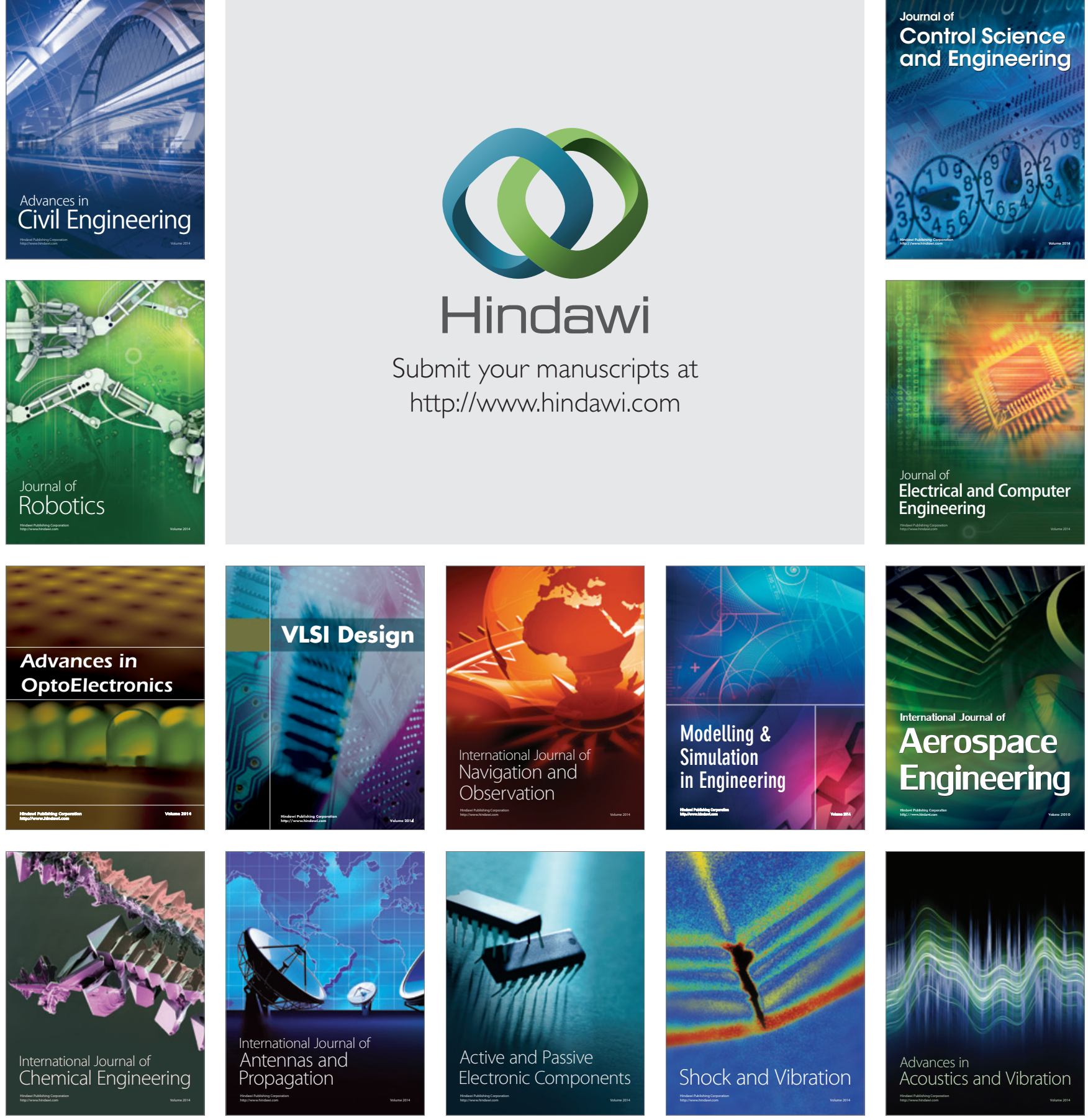